\title{
Multiple Length and Time-scale Approaches in Materials Modeling
}

\author{
Likun Tan \\ Thayer School of Engineering, Dartmouth College, New Hampshire, USA
}

\section{Email address:}

Likun.Tan@dartmouth.edu

\section{To cite this article:}

Likun Tan. Multiple Length and Time-scale Approaches in Materials Modeling. Advances in Materials. Special Issue: Advances in Multiscale Modeling Approach. Vol. 6, No. 1-1, 2017, pp. 1-9. doi: 10.11648/j.am.s.2017060101.11

Received: August 4, 2016; Accepted: August 21, 2016; Published: September 3, 2016

\begin{abstract}
Multiscale modeling has become an essential tool in understanding and designing materials and physical systems with characteristics at multiple length and time scales. Although modern computational techniques are able to track the material behaviors from the nano-scale atomic vibrations at femtoseconds to the macroscopic plastic deformations of metals at seconds, simulations of physical phenomena of engineering interest are often limited by overwhelming computation time. The objective of multiscale methods is to predict the important physical behaviors without resolving the full details of the system, through averaging/coarse-graining the structure in length and/or extracting the slow time-scale dynamics. This paper reviews the state-of-the-art multiscale methods with applications in material science and biological systems.
\end{abstract}

Keywords: Multiscale Modeling, Metals, Composites, Biological Molecules

\section{Introduction}

Multiscale modeling and simulation is a rapidly developing field of research over the last three decades. Even though mathematicians have long studied multiscale problems, the profound significance of this topic has been drawn attraction in applied sciences and engineering, in particular, material science, mechanics sciences, and biology. Materials and systems can often exhibit different features at different scales, in terms of length and/or time. For example, shape memory alloys with heterogeneous microstructure may show distinct hysteresis loop compared [2, 10, 12]; Folding time for the proteins is approximately on the order of seconds while the vibration of the covalent bolds is at femtoseconds $[34,52]$; The deformation of metal is governed by continuum mechanics under a typical loading rate of $10^{-3}-10^{0} \mathrm{~s}$ but the nano-scale phenomena follows the Schrodinger's equation in quantum mechanics with a characteristic time scale of attoseconds [42].

We use 'fine-scale' and 'coarse-scale' to refer to the physics at the two distinct scales. Here, fine-scale problems refer to the behaviors at the quantum or atomic level with a characteristic time scale between attoseconds and femtoseconds, while coarse-scale problems stand for the mesoscopic or macroscopic phenomena with a typical time scale between microseconds to seconds. For many practical problems, it is simply impossible to represent or resolve the full details of the fine-scale problem due to the overwhelming computational costs. Therefore we must seek alternative approaches that are more efficient and affordable. The computational techniques aimed at efficient representation or solution of such fine-scale problems. The approaches for multiscale modeling can be classified into 'top-down' and 'bottom-up' [61]. The top-down approach involves solving the fine-scale phenomena from the coarsescale behavior, while the bottom-up approach aims at obtaining the coarse/effective behavior given the physical governing laws at the fine scale. According to the nature of the problem, multiscale methods can be also categorized into multiple length-scale and multiple time-scale approaches. Engineers are often interested in the characteristic material properties and biological processes at the mesoscopic level, but lack a general theory for describing such problems. It is reasonable to assume that coarse scale behavior is affected by the underlying physics at the fine scale. For example, it is well recognized that the mesoscopically observed plastic behavior of metal is due to dislocation creation, propagation and accumulation at the atomic scale; Protein folding that happens on the order of seconds is influenced by the vibration of covalent bonds at femtoseconds. For engineering 
and biological applications, it is important to develop reliable yet affordable scale transition methods in which a multiscale modeling approach would make a difference.

This paper will focus on discussing a class of bottom-up approaches that present the state-of the-art, which is followed by the applications of multiscale approaches on some of the problems in material sciences and biological process.

\section{Multiple Length-scale Approaches}

\subsection{Mathematical Homogenization}

Mathematical homogenization is a technique of retrieving a homogenous (or effective) equation for a partial differential equation with highly oscillatory coefficients [43, 44, 51]. Consider the problem

$$
\frac{\partial u^{\varepsilon}}{\partial t}=\nabla \cdot\left(a\left(x, \frac{x}{\varepsilon}\right) \nabla u^{\varepsilon}(x, t)\right), \quad x \in \Omega,
$$

with the boundary condition $\left.u^{\varepsilon}\right|_{\partial \Omega}=0$. In this problem (1), the multiscale nature comes from the coefficients $a(x, x / \mathcal{E})$, which contain two scales: a fine scale of $O(\varepsilon)$ and a coarse scale of $O(1)$. This two-scale feature can be used to describe many important physical processes such as heat conduction and phase transformation in a composite material. Denote $y=x / \varepsilon$ and assume that $a(x, y)$ is periodic in $y$ in an idealized setting. Following [44], $u^{\varepsilon}$ can be expanded using asymptotic expansion as

$$
u^{\varepsilon}(x, t)=u_{0}(x, t)+\varepsilon u_{1}\left(x, \frac{x}{\varepsilon}, t\right)+O\left(\varepsilon^{2}\right),
$$

where $\varepsilon<<1$ and $u_{0}$ is normally the homogenized solution that satisfies

$$
\frac{\partial u_{0}}{\partial t}=\nabla \cdot\left(A(x) \nabla u_{0}(x, t)\right) \quad \text { in } \Omega,
$$

and the boundary condition $\left.u_{0}\right|_{\partial \Omega}=0$. Note that the fine scale variables no longer exist in (3) and $A(x)$ may be thought of as being the effective coefficient presenting the overall properties of the system on the coarse scale of $O(1)$. Determining $A(x)$ often requires solving a unit-cell problem. In the one-dimensional case, $A(x)$ is simply given by the harmonic average, i.e.

$$
A(x)=\left(\int_{0}^{1} \frac{1}{a(x, y)} d y\right)^{-1} .
$$

\subsection{Cohesive Zone Model}

Cohesive zone model (CZM) [45] is a finite element-based computational model in the field of fracture mechanics to determine the possibility of crack extension and the direction of crack growth and branching. The crack takes place across an extended crack tip, or cohesive zone. One of the main aspects of CZM is to study the fracture of crystalline materials by modeling the microstructure as grains and grain boundaries. The constitutive equations of grains and grain boundaries take different forms due to their distinct deformation mechanisms. The grain boundary model requires evolutionary equations taking into account micromechanical physical phenomena such as grain boundary sliding, grain boundary cavitation, plus grain boundary embrittlement. For example, in high temperature creep rupture, the constitutive law for grains is taken to be

$$
\begin{aligned}
& \dot{\varepsilon}_{i j}=\dot{\varepsilon}_{i j}^{e}+\dot{\varepsilon}_{i j}^{C}, \\
& \dot{\varepsilon}_{i j}^{e}=\frac{1+v}{E} \dot{\sigma}_{i j}-\frac{v}{E} \dot{\sigma}_{k k} \delta_{i j}, \\
& \dot{\varepsilon}_{i j}^{C}=\frac{3}{2} B \sigma_{e}^{n} \frac{s_{i j}}{\sigma_{e}},
\end{aligned}
$$

where $\dot{\varepsilon}_{i j}$ is the total strain rate, $\dot{\varepsilon}_{i j}^{e}$ is the elastic part and $\dot{\varepsilon}_{i j}^{C}$ is the creep strain rate; $\sigma$ is the stress tensor, $E$ and $v$ are respectively the Young's modulus and Poisson's ratio; $s_{i j}$ and $\sigma_{e}$ are respectively the deviatoric stress tensor and equivalent stress; and $B$ and $n$ are creep parameters. The constitutive equation for grain boundary composes of two parts. One is due to grain boundary separation given by

$$
\delta=\delta_{c}+\delta_{b} H\left(\sigma_{n}-\sigma^{*}\right)
$$

where $\delta_{c}$ is the opening displacement due to void nucleation and growth, and $\delta_{b}$ can be roughly attributed to the decohesion of the grain boundaries between cavities and is activated when the normal stress $\sigma_{n}$ reaches a critical value $\sigma^{*}$, and $H$ is the Heaviside function. The second contribution is grain boundary sliding along the surface. A popular model assumes the grain boundary as a Newtonian fluid,

$$
\begin{aligned}
& \dot{u}_{1}=\tau_{1} / \eta_{b}, \\
& \dot{u}_{2}=\tau_{2} / \eta_{b},
\end{aligned}
$$

where $\dot{u}_{1}$ and $\dot{u}_{2}$ are the sliding rates in the two tangent directions; $\tau_{1}$ and $\tau_{2}$ are the corresponding interfacial shear tractions, and $\eta_{b}$ is the grain boundary viscosity.

CZM works for any physical materials given appropriate material models. It is easily to implement in commercial finite element software since it follows the standard formulation in continuum mechanics.

\subsection{Quasi-continuum Method}

Quasi-continuum (QC) method analyzes the mechanical deformation of crystalline solids based on atomic models. In 
the continuum theory of nonlinear elasticity, the governing equation for linear momentum balance takes an equivalent form as a variational problem,

$$
\min _{u} E(u)=\int_{\Omega} f(\nabla u) d x,
$$

where $u$ and $E$ denote the displacement field and the total elastic energy, respectively; $f$ is the stored energy functional, dependent on the external loading or boundary conditions. In practice empirical methods are often adopted to determine $f$. QC method was proposed in [55] for the analysis of crystalline materials, in which the fine-scale model is taken as the molecular mechanics of atoms that make up the crystals. The coarse-scale problem is assumed to be at the macroscopic scale in which finite element method is applied. The material consists of finite element triangle elements with standard continuous piecewise-linear basis functions. Therefore, $\nabla u$ is constant within each element. Let $K$ denote the finite element and $E_{K}(u)$ be the elastic energy in $K$, which depends on the constant deformation gradient $\left.\nabla u\right|_{K}$ within this element. Therefore, the total energy in the QC setting becomes

$$
\bar{E}(u)=\sum_{K} n_{K} E_{K}(u)
$$

where $n_{K}$ is the number of unit cells in $K$. This approach provides a general framework for estimating $f$ rater than treating it empirically. Note that the effective property $f$ on the coarse scale can be computed on the fly using fine-scale models from molecular mechanics.

\section{Multiple Length-Scale Approaches}

\subsection{Center Manifold Theory}

The center manifold theory was developed by Carr et al. $[13,14]$, which is applied to an autonomous system of ordinary differential equations (ODEs) with the general form,

$$
\begin{aligned}
& \frac{d x}{d t}=A x+f(x, y), \\
& \frac{d y}{d t}=B y+g(x, y),
\end{aligned}
$$

where $x$ and $y$ are $n$ - and $m$ - dimensional vectors, respectively; $A$ and $B$ are constant square matrices where the real parts of eigenvalues of $A$ are all zero while the real parts are all negative for $B$; and finally, $f$ and $g$ are simply non-zero functions of variables $x$ and $y$ which are at least $C^{2}$ continuous. Let $S$ denote a set of solutions of this system $(x(t), y(t))$ for $t>0$. The set $S$ is a local invariant manifold if any solution of this system starting from an initial condition $(x(0), y(0))$ stays within this set for a finite period of time $T$. The set $S$ is called an invariant manifold if $T \rightarrow \infty$. The dynamics of the variables $x$ and $y$ can vary fundamentally determined by the eigenvalues - $x$ follow a more or less monotonic trend and can be regard as the coarse (i.e. retained) variables, while $y$ behave in an oscillatory mode and are the fine variables. Moreover, the fine variables, $y$, can be parameterized on the coarse phase space via $y=h(x)$, which is called a center manifold if $h(0)=0, h^{\prime}(0)=0$, and $y$ is an invariant manifold for $h$ is smooth. The coarse-scale problem becomes a $n$ dimensional dynamical system, which presents the flow $u$, on the center manifold,

$$
\frac{d u}{d t}=A u+f(u, h(u))
$$

By substituting $y(t)=h(x(t))$ into the second equation of (10), we obtain a (usually nonlinear) system of partial differential equations,

$$
\frac{d u}{d t}[A x+f(x, h(x))]=B h(x)+g(x, h(x)),
$$

together with the initial conditions $h(0)=0, h^{\prime}(0)=0$, formulate the governing equations for the fine variables (i.e. center manifold). In general there are more than one center manifold in (10), therefore, $h$ is not uniquely determined by $\mathrm{x}$. This requires sophisticated numerical algorithms to solve (12).

\subsection{Young Measure Approach}

The Young Measure approach was first proposed by Artstein et al. [4] with the aim to examine the limit behavior of the following system of ordinary differential equations

$$
\begin{aligned}
& \frac{1}{\varepsilon} \frac{d x}{d t}=f(x, y), \\
& \frac{d y}{d t}=g(x, y),
\end{aligned}
$$

where $\varepsilon \rightarrow 0$. (13) is called a slow-fast system in which explicit separation of time scales exist with $x$ and $y$ are the coarse and fine variables, respectively. The slow-fast system is often written on the slow time scale, $s:=\varepsilon t$, as

$$
\begin{aligned}
& \frac{d x}{d s}=f(x, y), \\
& \varepsilon \frac{d y}{d s}=g(x, y),
\end{aligned}
$$

In the classical approach, for example, the Tikhonov approach [59], the limit dynamics has been shown to converge to a system of differential algebraic equations (DAEs) 


$$
\begin{aligned}
& \frac{d x}{d s}=f(x, y), \\
& 0=g(x, y),
\end{aligned}
$$

However, the Young measure theory [4] shows that the fast dynamics converges to an invariant (probability) measure that is drifted by the slow dynamics. More generally, for any continuous function $F$,

$$
F\left(y^{\varepsilon, x, y_{0}}(s)\right) \rightarrow \int_{R^{n}} F(r) \mu_{s, x, y_{0}}(r) d r
$$

where $y^{\varepsilon, x, y_{0}}(s)$ denotes the solutions of the fast system at the slow time $s$ with $x$ fixed and $\mu_{s, x, y_{0}}$ is the invariant measure (i.e. Young measure) that can be approximated numerically as averages of $M$ Dirac masses at $M$ values of $y$, i.e.

$$
\mu_{s, x, y_{0}} \approx \frac{1}{M} \sum_{i=1}^{M} \delta\left(y^{(0), x(s), y_{0}}\left(t_{i}\right)\right),
$$

where $y^{(0), x(s), y_{0}}$ is the fast flow on the fast time scale and $t_{i}$ is the discrete time interval in the fast run with initial condition $y_{0}$ and the coarse variables $x$ hold constant. Normally $M$ has to be large enough for the fast flow to converge to an asymptotically stable attractor.

Averaging of the coupled slow and fast motions were studied in $[5,9]$. The non-autonomous case (i.e. the righthand-side vector of the fast system is also a function of time) was investigated in $[4,8]$. For the dynamical systems without explicit separation of time scales, a rigorous theory was given in [7] and the application of this theory to a discrete KDVBurgers type equation was performed in [6].

\subsection{Macro-Micro Filtering}

The problems considered in [24, 25, 32] are stochastic differential equations with multiscale features given as,

$$
\begin{aligned}
& \frac{d x}{d t}=f(x, y)+\sigma_{x} \dot{W}_{x}(t), \\
& \frac{d y}{d t}=\frac{1}{\varepsilon} g(x, y)+\frac{\sigma_{y}}{\sqrt{\varepsilon}} \dot{W}_{y}(t), \\
& v_{m}=x_{m}+\sigma_{m}^{0},
\end{aligned}
$$

where $x$ and $y$ present the coarse- and fine-scale variables, respectively, with distinct time scales characterized by small $\varepsilon ; \sigma$ and $\dot{W}$ denote the noise strength and white noise in time, respectively; $v_{m}$ denotes the noisy observation of $x_{m}=x\left(t_{m}\right)$ at discrete observation time interval (i.e. coarse time scale), $\Delta t=t_{m+1}-t_{m}$ with $m$ being the coarse time step, and $\sigma_{m}^{0}$ is the Gaussian noise. Moreover, let $\delta t$ denote the fine time step.

The dynamical system (18) has no clear separation of timescales. In this case, the fine-scale dynamics is coupled with the coarse model directly and the fine model retained a memory of its initial conditions, therefore, fine-scale solutions can be obtained from running the fast flow equation while freezing coarse-scale variables as in the Young measure theory and PTA. The macro-micro filtering (MMF) algorithm consists of two parts: a 'macro-filter' that deals with the coarse-scale problem with partial information from fine-scale model and a 'micro-filter' that essentially solves an inverse problem for matching the fine variables with parameterized coarse variables. The aim of MMF is to generate a posterior ensemble of $\left\{x_{k, m+1}^{+}, y_{k, m+1}^{+}, k=1, \cdots, K\right\}$, given a posterior ensemble $\left\{x_{k, m}^{+}, y_{k, m}^{+}, k=1, \cdots, K\right\}$ at previous time step, where $K$ denotes the ensemble size; Here, the superscript ' + ' and ''indicate prior and posterior variables before and after filtering, respectively. The procedure of MMF is as follows:

1. Run the fine dynamics of (18) with initial conditions $y_{k, m}^{+}$to generate a fine-scale prior ensemble $\left\{y_{k, m+N \delta t}^{-}, k=1, \cdots, K\right\}$ while fixing the coarse variable at $x=x_{k, m}^{+}$, where $N$ is the number of total fine steps and $N \delta t<\Delta t$ to guarantee computational efficiency.

2. Compute the time average of the prior ensemble of fine variables, i.e.

$$
\bar{y}_{m+N \delta t}^{-}=K^{-1} \sum_{k=1}^{K} y_{k, m+N \delta t}^{-} .
$$

3. Obtain the prior ensemble of coarse variables $x_{k, m+1}^{-}$at the next coarse time step (the 'macro-filter') by

$$
x_{k, m+1}^{-}=x_{k, m}^{+}+\frac{d x}{d t} \Delta t
$$

where $d x / d t$ can be approximated from the previous result $y=\bar{y}_{m+N \delta t}^{-}$.

4. Estimate the posterior ensemble $x_{k, m+1}^{+}$using ensemblebased filtering techniques.

5. Determine the posterior ensemble of the fine variables $y_{k, m+1}^{+}$(the 'micro-filter') by solving the inverse problem,

$$
\min _{y}\left\|x_{m+1}^{+}-h(y)\right\|_{R_{m+1}^{+}}^{2}
$$

From the timesaving's point of view, besides the requirement of $N \delta t<\Delta t$ as in PTA, the computational cost of the minimization solver has to be taken into account.

\section{Multiscale Modeling in Length and Time}

\subsection{Parameterized Locally Invariant Manifolds}

The method of Parameterized Locally Invariant Manifolds 
(PLIM) [3] stems from invariant manifold theory [33, 39] and averaging theorem [21,60]. This method works for autonomous dynamical systems without explicit separation of time-scales (i.e., no distinction of fine or coarse variables in the system) in the general form

$$
\frac{d f}{d t}(t)=H(f(t)), \quad f(0)=f_{*},
$$

where $f$ is a vector field of variables and $H$ is a generally nonlinear function of $f$. The dynamical system (22) is the fine-scale problem where $f$ is called the fine variables. The novelty of PLIM lies in that the coarse variables can be defined as arbitrary time-averaged functions of fine-scale variables rather than instantaneous state functions based on the practical interest. Similar to the center manifold theory, the fine variables are computed and presented as local invariant manifolds via parameterization on the coarse phase space. Coarse variables are defined as

$$
c(t):=\frac{1}{\tau} \int_{t}^{t+\tau} \Lambda(f(p)) d p
$$

where $\tau$ is the period of time averaging. Consequently, an augmented ODE system is derived as

$$
\begin{aligned}
& \frac{d f_{f}}{d t}(t)=H\left(f_{f}(t)\right), \quad f_{f}(0)=f_{* *}, \\
& \frac{d f}{d t}(t)=H(f(t)), \quad f(0)=f_{*}, \\
& \frac{d c}{d t}(t)=\frac{1}{\tau}\left[\Lambda\left(f_{f}(t)\right)-\Lambda(f(t))\right], \quad c(0)=c_{*},
\end{aligned}
$$

where $f_{f}$ denote the augmented fine variables and $f_{f}(t):=f(t+\tau)$. The fine variables $f$ and $f_{f}$ are parameterized on the coarse space as $f:=G(c)$ and $f_{f}:=G_{f}(c)$. The governing equations for solving the invariant manifolds are

$$
\begin{aligned}
& \frac{\partial G_{f}}{\partial c}\left(\frac{1}{\tau}\left[\Lambda\left(G_{f}\right)-\Lambda(G)\right]\right)=H\left(G_{f}\right), \\
& \frac{\partial G}{\partial c}\left(\frac{1}{\tau}\left[\Lambda\left(G_{f}\right)-\Lambda(G)\right]\right)=H(G),
\end{aligned}
$$

by assuming $G=f$ and $G_{f}=f_{f}$ and substituting in (24). The dynamical behavior of coarse variables can be computed by

$$
\frac{d c}{d t}=\frac{1}{\tau}\left[\Lambda\left(G_{f}(c)\right)-\Lambda(G(c))\right]
$$

The method of PLIM allows more freedom to select coarse variables and the characteristic timescales of coarse dynamics compared with the center manifold theory. However, non-uniqueness of the local invariant manifolds remains an issue and needs to be treated with care. One thing to note is that the coarse variables, $c$, can exhibit dynamical behavior on a coarser length scale when $\Lambda$ is taken as the spatial average of the fine states $f$, therefore, PLIM predicts a dynamical behavior (26) that can be coarser both in length and time.

\subsection{Practical Time Averaging}

The problems of interest here are slow-fast ODE systems (13). While Young measure theory provides us with the limit dynamics of fast flow (i.e. fine variables), the approach of Practical Time Averaging (PTA) $[57,58]$ is able to estimate the evolution of time averaged phase functions of the fast flow on the slow time scale. PTA can be regarded as a coupling between the Young measure theory and generalized method of averaging While the Young measure theory shows that the fast flow may not converge to an equilibrium of the standard DAE system as given in the Tikhonov approach, the idea of PTA is to check whether the limit dynamics of the time averages of the fast flow or the functions of the fast flow converges to the DAE of the Tikhonov method. The coarse variables and their evolution are written as

$$
\begin{aligned}
& c(s):=\frac{1}{\tau} \int_{s}^{s+\tau} \Lambda(y(p)) d p, \\
& \frac{d c}{d s}(s)=\frac{1}{\tau}[\Lambda(y(s+\tau))-\Lambda(y(s))],
\end{aligned}
$$

where $\tau$ is the period of time average. Generate the sequence on $\varepsilon$ of smooth functions $c^{\varepsilon, y_{0}}$ and the coarse evolution equation (27)2 becomes

$$
\begin{aligned}
\frac{d c^{\varepsilon, y_{0}}}{d s}(s)= & \frac{1}{\tau}\left[\Lambda\left(y^{\varepsilon, x(s+\tau), y_{0}}(s+\tau)\right)\right. \\
& \left.-\Lambda\left(y^{\varepsilon, x(s), y_{0}}(s)\right)\right],
\end{aligned}
$$

Taking the limit on both sides of (28) using the result from the Young measure theory (16) leads to

$$
\begin{aligned}
\frac{d \bar{c}^{y_{0}}}{d s}(s)= & \frac{1}{\tau}\left[\int_{R^{n}} \Lambda(r) \mu_{s+\tau, x(s+\tau), y_{0}}(r) d r\right. \\
& \left.-\int_{R^{n}} \Lambda(r) \mu_{s, x(s), y_{0}}(r) d r\right]
\end{aligned}
$$

where $\bar{c}^{y_{0}}$ denotes the weak limit of the sequence of the coarse variables $c^{\varepsilon, y_{0}}$ and is the variable of interest. PTA involves the following steps to solve the coarse-scale problem (29):

1. Approximate the right-hand-side vectors of the coarse theory (4.8) using (17), i.e. 


$$
\begin{aligned}
& \int_{R^{n}} \Lambda(r) \mu_{s, x(s), y_{0}}(r) d r \approx \frac{1}{M} \sum_{i=1}^{M} \Lambda\left(y^{(0), x(s), y_{0}}\right), \\
& \int_{R^{n}} \Lambda(r) \mu_{s+\tau, x(s+\tau), y_{0}}(r) d r \approx \frac{1}{M} \sum_{i=1}^{M} \Lambda\left(y^{(0), x(s+\tau), y_{0}}\right) .
\end{aligned}
$$

2. Compute coarse variable at the next coarse step $s+T$ using the Forward Euler scheme, i.e.

$$
\begin{aligned}
& c(s+T)=c(s)+\frac{T}{\tau}\left[\int_{R^{n}} \Lambda(r) \mu_{s+\tau, x(s+\tau), y_{0}}(r) d r\right. \\
&\left.-\int_{R^{n}} \Lambda(r) \mu_{s, x(s), y_{0}}(r) d r\right], \quad T>\tau .
\end{aligned}
$$

3. Start from new initial conditions $y_{0}$ that match $x$ and $c$ at $s+T$.

4. Repeat.

The main difficulty in PTA is the non-uniqueness of fine states $y_{0}$ that correspond to the coarse scale variables $x$ and $c$. An optimization step is often applied to select the nearest fine state from the previous one. As in PLIM, the coarse variable can be defined as the spatial and time averaged functions of the fine states.

\subsection{Heterogeneous Multiscale Modeling}

Heterogeneous Multiscale Method (HMM) [1, 50, 61, 62] is a general framework for multi-scale modeling. The term 'heterogeneous' is used to emphasize that the models at different scales may be of different nature, e.g. molecular dynamics at the fine scale and continuum mechanics at the coarse scale (note that in the original papers of HMM, fine scale and coarse scale are termed as micro scale and macro scale, respectively). The main idea is to solve the preconceived coarse-scale model with missing information obtained from the fine-scale model. Suppose the fine-scale system takes the form

$$
f(u, d)=0,
$$

where $u$ is the state variable and $d$ is the data needed for the set-up of the fine-scale problem.

The coarse-scale model is taken to be

$$
F(U, D)=0,
$$

where $U$ is the coarse state of the system, and $D$ stands for the missing component to be obtained from (a part of) the fine system (32). The procedure is as follows:

1. Solve the coarse model (33).

2. Estimate the missing data $D$ by running a number of constrained fine simulations. The constraint is imposed to make sure the coarse data $D$ and fine-scale variables are consistent, i.e., $d=d(U)$. Usually ergodicity assumption and an explicit separation of timescales are needed.

3. Solve the fine-scale model (32) to generate the missing coarse-scale data $U$.

Compared with other multiscale approaches (e.g. PLIM and PTA) that apply for dynamical systems with multiple time scales, HMM provides a more general strategy for a variety of problems in which multiscale modeling is desirable, for example, stochastic simulation algorithms with disparate rates and elliptic partial differential equations with multiscale data. Note that the multiple time-scale approaches discussed in Section 3 can fit into the framework of HMM, where capturing the coarse-scale behavior from fine dynamics is still the most challenging part.

In practice, one needs to explore as much as possible prior knowledge about both the coarse-scale and fine-scale models and make use of any features of the physical problems (e.g. time-scale separation, self-similarity etc.).

\subsection{Markov State Models}

The Markov state models (MSMs) [40, 53] deal with various problems in molecular dynamics (MD) simulations of biomolecules and crystalline materials. The main idea is to partition the conformational space into discrete sub-state, identify the kinetically relevant conformational states and the rates of interconversion between these states. Essential structural and dynamical properties of interest are based on the discrete sub-states. Some assumptions are required: one is that the transitions between the states in the partitions are memoryless, that is, the dynamics of the system jumping into the next state depends on the current state only, but is stochastic; the second assumption is that the molecular dynamics converges to a unique invariant measure (i.e. ergodicity property); and finally, the states are metastable such that typical trajectories remain within a region for sufficient long periods of time before transiting into another metastable state.

MSMs aim to approximate the effective dynamics that described by the transition statistics between metastable states and to qualitatively understand the multiple time- and length-scale behaviors beyond which are accessible by direct MD simulations. Significant work needs to be done to accurately predict the kinetics, identify the optimal partition of the conformational spaces and relate MSMs to experimental data.

\section{Applications and Future Challenges}

Multiscale methods have a wide range of applications in material science. Many materials have the heterogeneous structures in nature or by fabrication, such as cement and concrete [37, 46], crystalline alloys [18, 36], bulk metallic glasses [17, 29, 47, 48], shape memory composites [12, 20, 63] and reinforced polymers $[19,67,68]$. Cementitious materials can be modeled as lattice elements that consist of unhydrated cement and hydration products $[49,56]$; Cohesive zone model has been extensively applied to investigate the failure mechanism of alloys [26-28, 66, 69]; Modern homogenization techniques have been developed to study random composites 
$[15,35,38]$ as well as heterogeneous materials such as biological tissues [30] and Neo-Hookean-type composites [23, $54,65]$ with large deformations.

Another application area of multiscale methods is in predicting and understanding the long time-scale behavior of bio-molecular systems such as DNA and proteins. DNA has the multiscale structure in nature with all the scales relevant for its functioning, ranging from full details of atomistic interactions to coarse grained representations of collections of hundreds of atoms as a few spherical beads connected by worm -like chain springs. Various coarse-grained models are developed to study structural properties such as helical pitch, torsion and flexibility $[11,16,22]$, as well as the dynamical properties such as DNA binding [31, 64, 70-73]. Protein composes of various types of amino acids and shows complex features in structure, dynamics and interactions. Understanding the protein folding mechanism would be the main reason for developing coarse-grained methods, as it plays an essential functional role in living cells. One of the major challenge is to characterize the equilibrium ensemble of folding pathways and their relative probability. The Markov state model [41] provides an efficient way to extract the full ensemble of transition pathways via partitioning the state spaces into sub-level conformational spaces.

Multiscale methods have been developed for over three decades and have been shown successful applications in a variety of fields, however, challenges still remain. One of the major issues is the reliability of the coarse-scale model. The fine-scale model is normally well defined with detailed information of the physical problems but requires a huge amount of computational times. Most multi-scale approaches rely on building up the coarse model with missing components that need to be obtained from the fine-scale model. The methods such as CZM and HMM require an appropriate coarse model to retrieve effective physical behavior at the coarse scale. Another challenge is the linking between the fine-scale data and the coarse-scale parameters. It is easier to compute the coarse variables given fine-scale data, however, in some cases, initial fine-scale data are required given coarse variables at a fixed slow time, for example, in PTA and HMM, which can be difficult due to non-uniqueness of fine solutions. Sophisticated techniques are needed to deal with this issue. Finally, better validation procedure of the coarse-scale physics is needed, which requires more advanced experimental tools.

\section{Acknowledgement}

This work was supported in part by NIH grant R01 EB018230 awarded by the National Institute of Biomedical Imaging and Bioengineering.

\section{References}

[1] Assyr Abdulle, E Weinan, Bjorn Engquist, and Eric VandenEijnden. The heterogeneous multiscale method. Acta Numerica, 21:1-87, 2012.
[2] Jacob Aboudi. The response of shape memory alloy composites. Smart materials and structures, 6(1):1, 1997.

[3] A. Acharya. Parametrized invariant manifolds: a recipe for multiscale modeling? Computer Methods in Applied Mechanics and Engineering, 194(27-29):3067-3089, 2005.

[4] Z. Artstein. Singularly perturbed ordinary differential equations with non-autonomous fast dynamics. Journal of Dynamics and Differential Equations, 11(2):297-318, 1999.

[5] Z. Artstein. On singularly perturbed ordinary differential equations with measure-valued limits. Mathematica Bohemica, 127(2):139-152, 2002.

[6] Z. Artstein, C. W. Gear, I. G. Kevrekidis, M. Slemrod, and E.S. Titi. Analysis and Computation of a discrete KDVBurgers type equation with fast dispersion and slow diffusion. Arxiv preprint arXiv:0908.2752, 2009.

[7] Z. Artstein, I.G. Kevrekidis, M. Slemrod, and E.S. Titi. Slow observables of singularly perturbed differential equations. Nonlinearity, 20:2463, 2007.

[8] Z. Artstein and M. Slemrod. On singularly perturbed retarded functional differential equations. Journal of Differential Equations, 171(1):88-109, 2001.

[9] Z. Artstein and A. Vigodner. Singularly perturbed ordinary differential equations with dynamic limits. In Proceedings of the Royal Society of Edinburgh-A-Mathematics, volume 126, pages 541-570. Cambridge Univ Press, 1996.

[10] K. Bhattacharya. Phase boundary propagation in a heterogeneous body. Proceedings: Mathematical, Physical and Engineering Sciences, pages 757-766, 1999.

[11] Yannick J Bomble and David A Case. Multiscale modeling of nucleic acids: insights into dna flexibility. Biopolymers, 89(9):722-731, 2008.

[12] James G Boyd and Dimitris C Lagoudas. Thermomechanical response of shape memory composites. Journal of intelligent material systems and structures, 5(3):333-346, 1994.

[13] J. Carr. Applications of centre manifold theory, volume 35. Springer, 1981.

[14] Jack Carr. Applications of centre manifold theory, volume 35. Springer Science \& Business Media, 2012.

[15] Pedro Ponte Castaneda. Second-order homogenization estimates for nonlinear composites incorporating field fluctuations: I-theory. Journal of the Mechanics and Physics of Solids, 50(4):737-757, 2002.

[16] Z Chen. Geometric nonlinearity and mechanical anisotropy in strained helical nanoribbons. Nanoscale, 6(16):9443-9447, 2014.

[17] Jiahao Cheng and Somnath Ghosh. Computational modeling of plastic deformation and shear banding in bulk metallic glasses. Computational Materials Science, 69:494-504, 2013.

[18] Jiahao Cheng and Somnath Ghosh. A crystal plasticity fe model for deformation with twin nucleation in magnesium alloys. International Journal of Plasticity, 67:148-170, 2015.

[19] FT Fisher, RD Bradshaw, and LC Brinson. Effects of nanotube waviness on the modulus of nanotube-reinforced polymers. Applied Physics Letters, 80(24):4647-4649, 2002. 
[20] Yasubumi Furuya, Atsushi Sasaki, and Minoru Taya. Enhanced mechanical properties of tini shape memory fiber/al matrix composite. Materials transactions, JIM, 34(3):224-227, 1993.

[21] John Guckenheimer and Philip J Holmes. Nonlinear oscillations, dynamical systems, and bifurcations of vector fields, volume 42. Springer Science \& Business Media, 2013.

[22] Qiaohang Guo, Zi Chen, Wei Li, Pinqiang Dai, Kun Ren, Junjie Lin, Larry A Taber, and Wenzhe Chen. Mechanics of tunable helices and geometric frustration in biomimetic seashells. EPL (Europhysics Letters), 105(6):64005, 2014.

[23] I Hariton, EA Socolsky, et al. Neo-hookean fiber-reinforced composites in finite elasticity. Journal of the Mechanics and Physics of Solids, 54(3):533-559, 2006.

[24] J. Harlim. Numerical strategies for filtering partially observed stiff stochastic differential equations. Journal of Computational Physics, 230(3):744-762, 2011.

[25] J. Harlim and AJ Majda. Mathematical strategies for filtering complex systems: Regularly spaced sparse observations. Journal of Computational Physics, 227(10):5304-5341, 2008.

[26] Zupan $\mathrm{Hu}$, Wei $\mathrm{Lu}$, and MD Thouless. Slip and wear at a corner with coulomb friction and an interfacial strength. Wear, 338:242-251, 2015.

[27] Zupan Hu, Wei Lu, MD Thouless, and JR Barber. Simulation of wear evolution using fictitious eigenstrains. Tribology International, 82:191-194, 2015.

[28] Zupan $\mathrm{Hu}$, Wei Lu, MD Thouless, and JR Barber. Effect of plastic deformation on the evolution of wear and local stress fields in fretting. International Journal of Solids and Structures, 82:1-8, 2016.

[29] Lu Huang, Chao Pu, Richard K Fisher, Deidra JH Mountain, Yanfei Gao, Peter K Liaw, Wei Zhang, and Wei He. A zr-based bulk metallic glass for future stent applications: Materials properties, finite element modeling, and in vitro human vascular cell response. Acta biomaterialia, 25:356-368, 2015.

[30] Jay D Humphrey. Cardiovascular solid mechanics: cells, tissues, and organs. Springer Science \& Business Media, 2013.

[31] Ming Ji, Likun Tan, Linda Jen-Jacobson, and Sunil Saxena. Insights into copper coordination in the ecori-dna complex by esr spectroscopy. Molecular physics, 112(24):3173-3182, 2014.

[32] E.L. Kang and J. Harlim. Filtering partially observed multiscale systems with heterogeneous multiscale methodsbased reduced climate models. Monthly Weather Review, 140(3):860-873, 2012.

[33] Dimitrios Karagiannis, Daniele Carnevale, and Alessandro Astolfi Invariant manifold based reduced-order observer design for nonlinear systems. IEEE Transactions on Automatic Control, 53(11):2602-2614, 2008.

[34] Tomas Lindahl. Instability and decay of the primary structure of dna. nature, 362(6422):709-715, 1993.

[35] Oscar Lopez-Pamies and Pedro Ponte Castaneda. Secondorder estimates for the macroscopic response and loss of ellipticity in porous rubbers at large deformations. Journal of elasticity, 76(3):247-287, 2004.

[36] FE Luborsky. Amorphous metallic alloys. Butterworth and Co
(Publishers): London, UK, 1983.

[37] P Kumar Mehta. Concrete. structure, properties and materials. 1986.

[38] Graeme W Milton. The theory of composites. The Theory of Composites, by Graeme W. Milton, pp. 748. ISBN 0521781256. Cambridge, UK: Cambridge University Press, May 2002., page 748, 2002.

[39] RG Muncaster. Invariant manifolds in mechanics I: The general construction of coarse theories from fine theories. Archive for Rational Mechanics and Analysis, 84(4):353-373, 1984.

[40] F. Noe, I. Horenko, C. Schutte, and J.C. Smith. Hierarchical analysis of conformational dynamics in biomolecules: Transition networks of metastable states. The Journal of chemical physics, 126:155102, 2007.

[41] F. Noe, C. Schutte, E. Vanden-Eijnden, L. Reich, and T.R. Weikl. Constructing the equilibrium ensemble of folding pathways from short off-equilibrium simulations. Proceedings of the National Academy of Sciences, 106(45):19011-19016, 2009.

[42] Laurent Nottale. Scale relativity, fractal space-time and quantum mechanics. Chaos, Solitons \& Fractals, 4(3):361388, 1994.

[43] N Panasenko and NS Bakhvalov. Homogenization: Averaging processes in periodic media: Mathematical problems in the mechanics of composite materials, 1989.

[44] G Papanicolau, A Bensoussan, and J-L Lions. Asymptotic analysis for periodic structures, volume 5. North Holland, 1978.

[45] Kyoungsoo Park and Glaucio H Paulino. Cohesive zone models: a critical review of traction separation relationships across fracture surfaces. Applied Mechanics Reviews, 64(6):060802, 2011.

[46] Treval Clifford Powers and Theodore Lucius Brownyard. Studies of the physical properties of hardened portland cement paste. In Journal Proceedings, volume 43, pages 101-132, 1946.

[47] Chao Pu. Failure simulations at multiple length scales in high temperature structural alloys. PhD Thesis, 2015.

[48] Chao Pu and Yanfei Gao. Crystal plasticity analysis of stress partitioning mechanisms and their microstructural dependence in advanced steels. Journal of Applied Mechanics, 82(3):031003, 2015.

[49] Zhiwei Qian. Multiscale modeling of fracture processes in cementitious materials. TU Delft, Delft University of Technology, 2012.

[50] W. Ren et al. Heterogeneous multiscale method for the modeling of complex fluids and micro-fluidics. Journal of Computational Physics, 204(1):1-26, 2005.

[51] Enrique Sanchez-Palencia. Non-homogeneous media and vibration theory. In Non-homogeneous media and vibration theory, volume 127, 1980.

[52] Daniel V Santi, Anne Norment, and Charles E Garrett. Covalent bond formation between a dnacytosine methyltransferase and dna containing 5-azacytosine. Proceedings of the National Academy of Sciences, 81(22):6993-6997, 1984. 
[53] C. Schutte, F. Noe, J. Lu, M. Sarich, and E. Vanden-Eijnden. Markov state models based on milestoning. J. Chem. Phys., 134(20):204105, 2011.

[54] Nuo Sheng, Mary C Boyce, David M Parks, GC Rutledge, JI Abes, and RE Cohen. Multiscale micromechanical modeling of polymer/clay nanocomposites and the effective clay particle. Polymer, 45(2):487-506, 2004.

[55] Ellad B Tadmor, Michael Ortiz, and Rob Phillips. Quasicontinuum analysis of defects in solids. Philosophical magazine A, 73(6):1529-1563, 1996.

[56] Li Kun Tan, Erik Schlangen, and Guang Ye. Simulation of failure in hydrating cement particles systems. In Key Engineering Materials, volume 348, pages 737-740. Trans Tech Publ, 2007.

[57] Likun Tan, Amit Acharya, and Kaushik Dayal. Coarse variables of autonomous ode systems and their evolution. Computer Methods in Applied Mechanics and Engineering, 253:199-218, 2013.

[58] Likun Tan, Amit Acharya, and Kaushik Dayal. Modeling of slow time-scale behavior of fast molecular dynamic systems. Journal of the Mechanics and Physics of Solids, 64:24-43, 2014.

[59] A.N. Tikhonov. Systems of differential equations containing small parameters in the derivatives. Matematicheskii Sbornik, 73(3):575-586, 1952.

[60] Ferdinand Verhulst and JA Sanders. Averaging methods in nonlinear dynamical systems. Applied Mathematical Sciences, 59, 1985.

[61] E. Weinan, B. Engquist, and Z. Huang. Heterogeneous multiscale method: a general methodology for multiscale modeling. Physical Review B, 67(9):092101, 2003.

[62] E Weinan, Bjorn Engquist, Xiantao Li, Weiqing Ren, and Eric Vanden-Eijnden. Heterogeneous multiscale methods: a review. Commun. Comput. Phys, 2(3):367-450, 2007.

[63] Tao Xie. Tunable polymer multi-shape memory effect. Nature, 464(7286):267-270, 2010.

[64] Zhongyu Yang, Michael R Kurpiewski, Ming Ji, Jacque E Townsend, Preeti Mehta, Linda Jen-Jacobson, and Sunil Saxena. Esr spectroscopy identifies inhibitory cu2+ sites in a dna-modifying enzyme to reveal determinants of catalytic specificity. Proceedings of the National Academy of Sciences, 109(17):E993-E1000, 2012.
[65] Julien Yvonnet and Q-C He. The reduced model multiscale method $(\mathrm{r} 3 \mathrm{~m})$ for the non-linear homogenization of hyperelastic media at finite strains. Journal of Computational Physics, 223(1):341-368, 2007.

[66] J Zhang, J Johnston, and Aditi Chattopadhyay. Physics-based multiscale damage criterion for fatigue crack prediction in aluminium alloy. Fatigue \& Fracture of Engineering Materials \& Structures, 37(2):119-131, 2014.

[67] Jinjun Zhang, Bonsung Koo, Yingtao Liu, Jin Zou, Aditi Chattopadhyay, and Lenore Dai. A novel statistical springbead based network model for self-sensing smart polymer materials. Smart Materials and Structures, 24(8):085022, 2015.

[68] Jinjun Zhang, Bonsung Koo, Nithya Subramanian, Yingtao Liu, and Aditi Chattopadhyay. An optimized cross-linked network model to simulate the linear elastic material response of a smart polymer. Journal of Intelligent Material Systems and Structures, page 1045389X15595292, 2015.

[69] Jinjun Zhang, Kuang Liu, Chuntao Luo, and Aditi Chattopadhyay. Crack initiation and fatigue life prediction on aluminum lug joints using statistical volume element-based multiscale modeling. Journal of Intelligent Material Systems and Structures, 24(17):2097-2109, 2013.

[70] Ji, Ming, Sharon Ruthstein, and Sunil Saxena. "Paramagnetic metal ions in pulsed ESR distance distribution measurements." Accounts of chemical research 47.2 (2013): 688-695.

[71] Yang, Zhongyu, Ming Ji, and Sunil Saxena. "Practical aspects of copper ion-based double electron electron resonance distance measurements." Applied Magnetic Resonance 39.4 (2010): 487-500.

[72] Ruthstein, Sharon, Ming Ji, Preeti Mehta, Linda Jen-Jacobson, and Sunil Saxena. "Sensitive $\mathrm{Cu} 2+-\mathrm{Cu} 2+$ Distance Measurements in a Protein-DNA Complex by DoubleQuantum Coherence ESR." The Journal of Physical Chemistry B 117, no. 20 (2013): 6227-6230.

[73] Ruthstein, Sharon, Ming Ji, Byong-kyu Shin, and Sunil Saxena. "A simple double quantum coherence ESR sequence that minimizes nuclear modulations in $\mathrm{Cu} 2+$-ion based distance measurements." Journal of Magnetic Resonance 257 (2015): 45-50. 